\title{
VIII.
}

\section{Zur Anatomie des Nasenrachenraumes ${ }^{1}$ ).}

\author{
Von Dr. R. Poelchen, \\ Volontairarzt an der chirurgisehen Klinik zu Königsberg i, pr.
}

(Hierzu Taf. III.)

Im Jahre 1885 veröffentlichte Tornwaldt eine Monographie "Ueber die Bedeutung der Bursa pharyngea für Erkrankung und Behandlung gewisser Nasenrachenraumkrankheiten", in der er zu beweisen suchte, dass bei Beurtheilung und Beschreibung der Krankheiten genannten Raumes ein Bestandtheil desseiben zu wenig Beachtung gefunden habe, nehmlich die von J. J. C. Meyer 1842 zuerst beschriebene und benannte Bursa pharyngea. In seiner Arbeit giebt Tornwaldt zuerst die Resultate der anatomischen Untersuchungen Meyer's, Luschka's, Tortual's, Ganghofner's u. A., beschreibt das durch die Rhinoscopia posterior gewonnene Bild der normalen Bursa und knüpft daran eine Pathologie dieses Gebildes, die er durch 25 Krankengeschichten illustrirt. Tornwaldt kommt zu dem Resultat, dass die Bursa pharyngea häufig der Sitz von Krankheiten im Nasenrachenraume sei.

Die Tornwaldt'sche Arbeit hat theils die Zustimmung der Fachgenossen erfahren, theils, und wohl grösseren Theils, hat man seine Befunde und Schlussfolgerungen nicht anerkannt, vor Allem hat man das Vorhandensein einer Bursa pharyngea, wie sie in Luschka's Arbeit über den Schlundkopf des Menschen abgebildet ist, bestritten. - Hätte sich Tornwaldt die Bursa ph. wirklich so vorgestellt, oder hätte er sie so beschrieben, wie Luschka sie abbildet, nehmlich als ein ziemlich selbständiges Organ, das in seiner Form auf der Luschka'schen Tafel grosse Aehnlichkeit mit dem Hodensack eines Stieres hat, so wäre ihm mit Recht opponirt worden; denn ich glaube

1) Nach einem am 18. October in der medicinischen Gesellschaft zu Danzig gehaltenen Vortrage. 


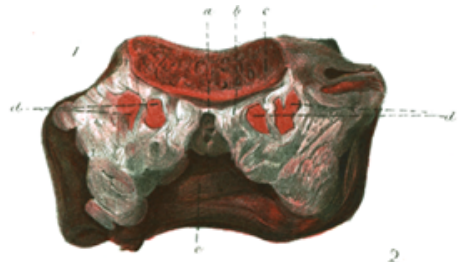

2

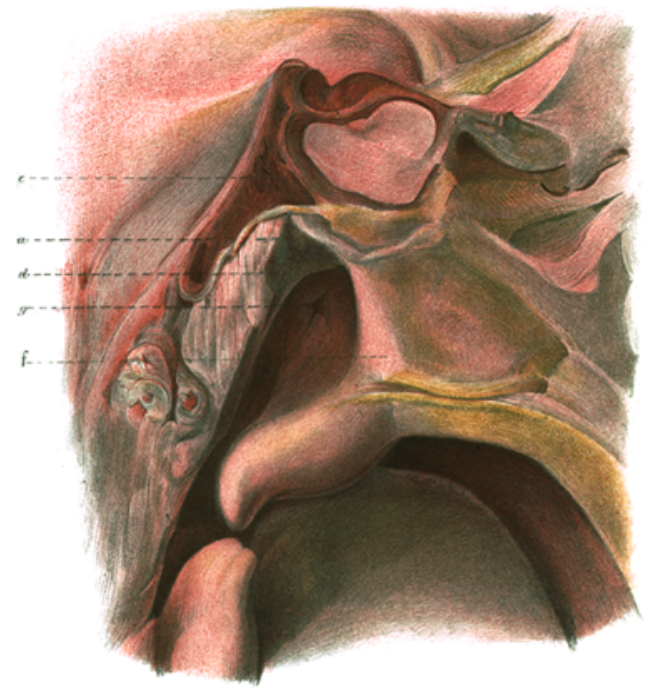

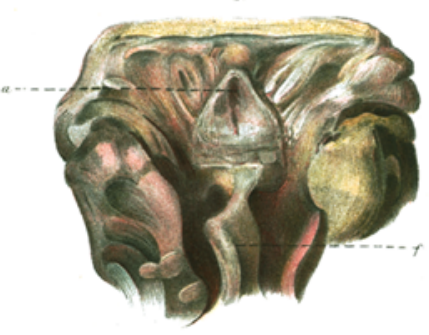

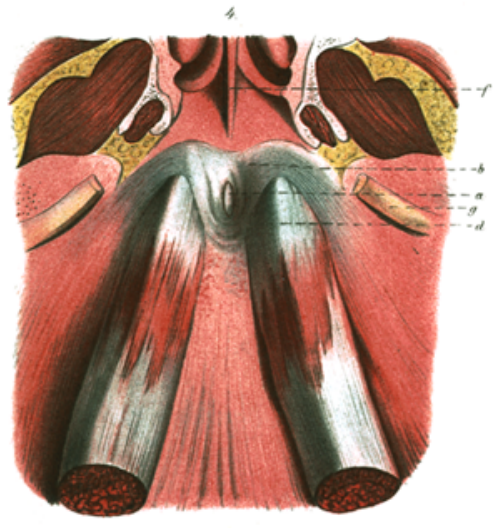


nicht, dass irgend jemand eimmal jenes Luschka'sche Organ zu Gesicht bekommen hat. Tornwaldt citirt nun aber weniger die Abbildung, als vielmehr die Beschreibung Luschka's; er sagt ausdrücklich, dass er sich nur auf seine spiegelbefunde stütze und sich begnügen müsse, die Bursa pharyngea so lange als Einsenkung der Schleimhat aufzufassen, bis durch anderweitige anatomische Untersuchungen eine etwaige andere Aufklärung gegeben sein wird.

Auf Tornwaldt's Anregung habe ich mich nun seit mehreren Jahren mit der Anatomie des Nasenrachenraumes beschäftigt und will hier kurz die Art und die Resultate meiner Untersuchungen mittheilen.

Macht man am Cadaver das Cavum pharyng. für das Auge dadurch zugängig, dass man, nach Herausuahme des Gehirns aus dem Schädel, diesen horizontal mit der Sägefläche auflagert und dann die Halsorgane nebst Zunge und weichem Gaumen entfernt, den Schlundkopf aber intact in situ lässt, so sieht man, nach gohöriger Reinigung mit starkem Wasserstrahl, in der Schleimhaut des Schlundkopfes, genau in der Medianlinie, ungefälır der Gegend des Tuberculum pharyng. entsprechend, bei sehr vielen Leichen ausgewachsener Individuen einen Spalt, ein Loch oder eine Grube; fühlt man mit dem Finger zu, so nimmt man dieser Grube entsprechend, bezw. bei ihrem Fehlen dicht vor dem Tub. pharyng. - eine von festen Rändern umgebene Vertiefung in den soliden Theilen des Schlundkopfdaches wahr. Diese Vertiefung geht nach unten, nach dem Brustkorbe zu, in eine Rinne ïber, welche zwischen den beiden $\mathrm{Mm}$. longi capitis (Rect. capt. antic. major.) gelegen ist und eine grösste Tiefe bis zu $1 \mathrm{~cm}$ erreicht. Bei Kindern regelmässig, auch nicht selten bei Individuen in den Jünglingsjahren, findet sich die Schleimhaut des Schlundkopfes sehr bedeutend verdickt durch starke Entwickelung des adenoiden Gewebes in der Mucosa, durch die sogen. Tonsilla pharyngea. Neugeborne zeigen in ihr meist 3 Spalten, von denen die mittlere wohl genau sagittal, die seitlichen oft in einem medianwärts concaven Bogen verlaufen, um hinten zusammenzutreffen. Neben diesen deutlicheren Spalten finden sich zahlreiche kleinere, die eine wesent- 
liche Bedeutung nicht haben, was ich übrigens auch von den 3 grösseren nicht annehme. In diesen, durch stärkere Entwickelung des adenoiden Gewebes ausgezeichneten Fällen ist der zufühlende Finger wohl kaum im Stande, von der Vertiefung unter der Schleimhaut etwas wahrzunehmen. Weitere Aufklärung über diese mittlere Gegend des in situ befindlichen Rachendaches zu gewinnen, scheint mir nicht möglich; man könnte ja noch sondiren, aber die Sonde ist in dem zarten Schleimhautgewebe ein sehr gefährliches Instrument, und möchte ich mich allen Sondenbefunden gegenüber sehr skeptisch verhalten. Will man weiter kommen, so muss man die Organe des Cavum pharyngo-nasale aus dem Schädel herausuehmen und dann weiter präpariren, vor allem Transversal- und Sagittalschnitte gefrorener Präparate anfertigen.

Für die Herausnahme des Nasenrachenraumes hat Klebs einen Trepan construirt, mit dem es sich ganz gut arbeitet, der aber wohl seines hohen Preises wegen wenig in Gebrauch genommen worden ist. Da der Klebs'sche Trepan aber noch einige andere Nachtheile hat, er es z. B. ausschliesst, die Grösse des zu gewinnenden Präparates beliebig zu bestimmen, so habe ich mich seit mehreren Jahren einer Methode bedient, die, wenn auch etwas roh, doch sehr einfach ist und mir für meine Zwecke wenigstens völlig genügt hat. Ich habe dieselbe mehrfach demonstrirt and wird nach ihr im Greifswalder pathologischanatomischen Institut seit 2 Jahren secirt. Sie wird folgendermaassen ausgeführt:

Der Scbädel bleibt in der oben angegebenen Lage fest auf einem Brette liegen. Indem die Schultern etwas erböht werden, wird die Halswirbelsäule in starke Lordose gebracht. Nun werden, wenn es nicht bereits vorher geschah, die Halsorgane derartig secirt, dass man den M. sternocleidomastoideus beiderseits fortnimmt, die Halsorgane dicht über dem Jugulum bis auf die Wirbelsäule quer durchtrennt und von letzterer bis zum 3. oder 2. Halswirbel sammt den Gefässen ablöst und nach dem Kinn hin überklappt. Ein $5-6 \mathrm{~cm}$ breites Stemmeisen setzt man auf den Körper des 2. oder 3. Halswirbels auf und stemmt senkrecht die Wirbelsäule und Schädelbasis durch, bis das Instrument in der hölzernen Unterlage festsitzt. Darauf werden die Verbindungen der Zunge mit dem Unterkiefer durehschnitten, und so der harte Gaumen zugänglich gemacht, auch dieser wird, möglichst nahe den Schneidezäbnen, durchstemmt mitsammt der Schädelbasis. In derselben Weise werden auch die seitlichen Verbindungen des Gaumens und Nasenrachen- 
raumes gelöst, nachdem vorher die etwa vorhandenen und störenden Oberkieferzähne extrabirt worden sind, und gelingt es nun unschwer, den Scblundkopf mit einem grösseren oder geringeren Theil der inneren Nase und den Halsorganen van der Schädelbasis her, nach Durchschneidung der Dura mater und der Nervi optici, berauszunehmen, ohne das Gesicht irgendwie verletzt zu haben.

Diese Sectionsmethode bietet folgende Vortheile: Sie ist sehr leicht und schnell auszuführen, sie gestattet die Untersuchung sämmtlicher Höhlen des Schädels, auch der Highmorshöhlen, wenn dieselben auch stets mitten durchgeschlagen werden, sie giebt die Möglichkeit, sich die obersten Enden der grossen Halsgefässe, den Verlauf der Carotis in den Knochen der Schädelbasis leicht zugängig zu machen. Um nun den $\mathrm{Na}$ senrachenraum gut übersehen zu können, empfiehlt es sich, die Halsorgane quer rom Schlunde abzuschneiden, den weichen, eventuell auch den harten Gaumen zu entfernen. Die an derartig gewonnenen Präparaten erhobenen pathologischen Befunde zeigen im Allgemeinen etwas recht Einförmiges, wären aber für den Rhino-Pharyngologen von erheblichem Interesse, ist doch in Bezug auf Anatomie und Pathologie des Nasenrachenranmes and der umliegenden Höhlen noch so vieles strittig.

Um in der uns beschäftigenden Untersuchang weiter $z u$ kommen, empfiehlt es sich, die ausgestemmten Präparate in einer Kältemischung gefrieren zu lassen und dann mit einer ganz feinen Säge in Transversal- und Sagittalschnitte zu zerlegen. Ein solcher Transversalschnitt findet sich in der Fig. 1 Taf. III abgebildet: Er zeigt zu oberst die quer durchschnittene rothe Masse der Pars basilaris ossis occipitis von vorn gesehen, darunter eine fibröse Substanz, die als stark verdicktes Periost den Knochen von unten deckt und den M. longus capitis jederseits in seine Fasermassen sich inseriren lässt. In der Mitte zeigt die fibröse Platte einen Defect, der von rother Schleimhaut ausgefüllt ist, so dass sich die Schleimhaut hier unmittelbar an den Knochen ansetzt. Dadurch, dass der M. Iongus cap. jederseits von dem Defect in der Massa fibrosa als ziemlich starker, von Schleimhaut bedeckter Wulst hervorspringt, wird eine auf dem Querschnitt schräg getroffene Rinne gebildet, deren Sohle, Rachenschleimhaut, sich wenige Millimeter vor dem Tuberculum pharyngeum in das fibröse Gewebe an der Unterfäche der Pars 
basilaris des Hinterhauptbeines "einbohrt", dasselbe auch ganz durchbohren kann and manchmal mehrere Millimeter noch in den Knochen selbst eindringt. Die Schleimhaut kann nun als dünne, einen offenen Sack oder Spalt formirende Haut an der genannten Stelle tief eindringen, und dann kann man ihr den $\mathrm{Na-}$ men Bursa oder Fossa pharyngea geben oder diese Stelle auch Recessus medius pharyngis nennen; oder die Schleimhaut ist durch adenoides Gewebe so verdickt, dass von einer Grube, einem Recessus nichts zu sehen ist, dafür findet man dann jenen Defect in der Massa fibrosa durch verdickte Schleimhaut ganz ausgefüllt, wie man eine Tasche mit doppelten Wänden dadurch ausfüllen kann, dass man den Raum zwischen den Wänden so lange wattirt, bis von der Taschenhöhlung nichts mehr zu sehen ist. Dieser Zustand fand sich theilweise an dem Präparat, das in der Fig. 1 abgebildet ist.

Fig. 2 stellt den Sagittalschnitt eines Ropfes dar, der ein ausgezeichnetes Specimen eines weit offenen, gegen $1 \frac{1}{2} \mathrm{~cm}$ tiefen Recessus medius darstellt, dessen Schleimhautwände einer irgendwie erheblicheren Entwickelung adenoiden Gewebes gänzlich entbehren. Diese Form ist es, die Tornwaldt besonders häufig am Lebenden gesehen haben will.

Fig. 3 zeigt das Dach des Nasenrachenraumes nach Entfernung der Schleimhaut und Muskelansätze. In der Mitte sieht man eine kahnförmige Grube, die wir bei erwachsenen Individuen durch die intacte Schleimhaut palpiren konnten. Sie zeigt in der Mitte ein Foramen coecum, das oft melarere Millimeter tief ist. Der hintere Theil der Grube ist besonders scharf umrandet und laufen die Ränder hinten im Tuberc. pharyngeum zusammen. Lateral von der kahnförmigen Grube, unmittelbar an ihre Ründer anstossend, findet sich je eine undeutlich dreiseitige unebene Fläche, die den Mm. longi capitis zum Ansatz dient. Durch jenes Foramen coecum dringt die Schleimhaut oft bis in die Substanz des Knochens.

Fig. 4 soll ein Gesammtbild des gegensuitigen Verhaltens der Halswirbelsäule, der $\mathrm{Mm}$. longi capitis und der Decke des Nasenrachenraumes geben, nachdem die Schleimhaut mit der Schlundmusculatur entfernt worden ist. Zwischen den auf dem Querschnitt dreieckigen Mm. longi capitis sieht man die oben 
erwähnte Rinne, welche in die kahnförmige Grube der Massa fibrosa der Schädelbasis übergeht. $Z u$ beiden Seiten des hinteren Theiles der kahnförmigen Grube heften sich die genannten Muskeln in dreiseitiger Fläche an die Schädelbasis. Auf diese dreiseitige Form des oberen Endes der Mm. longi capitis möchte ich ganz besonders hinweisen, sie trägt sehr wesentlich zur Formirung der Bursa pharyngea bei. Der Longus capitis sinister bildet hauptsächlich die so auffallend hohe Seitenwand des in Fig. 2 abgebildeten, in der Medianebene durchgesägten Recessus medius (Bursa pharyng.).

Die dreiseitige Querschnittsform des Longus capitis mit stark nach vorn vorspringendem Winkel an der Insertion scheint übrigens bisher übersehen worden zu sein, wenigstens konnte ich in der reichhaltigen Bibliothek der Königsberger anatomischen Anstalt keine Abbildung auffinden, in der dies Verhalten richtig dargestellt wäre. Hätte man die Muskeln etwas genauer auf ihre Ansatzform am Os occipitis angesehen, so hätte man auch wohl die an letzterem so häufig vorkommende kahnförmige Grube beschrieben, welche mit der in der Massa fibrosa befindlichen correspondirt. Die Fossa navicularis corporis ossis occipitis wird seitlich durch zwei Knochenleisten begrenzt, die dem Ansatzdreieck der Mm. longi cpt. zur medialen Begrenzung dienen und hinten im Tuberc. pharyng. zusammenlaufen. In der Mitte dieser kahnförmigen Grube des Knochens findet sich häufig, aber durchaus nicht immer, ein Foramen coecum, welches dem in der Massa fibrosa entspricht. Dasselbe ist als Fovea pharyngea schon mehrfach beschrieben worden, ist; wenn überhaupt vorhanden, sehr wechselnd in Grösse, Gestalt, Lage und Tiefe, reicht in einzelnen Fällen bis dicht an die Subst. compacta des Clivus Blumenbachii. Zur Feststellung dieser Details habe ich mit liebenswürdigst ertheilter Erlaubniss des Herrn Professor Stieda die 630 Schädel der hiesigen Anatomie untersucht: Es ergab sich, dass die erwähnten Grenzleisten nie vermisst wurden; eine deutliche, demonstrable kahnförmige Grube (Fossa navicularis) mit oder ohne Foramen coecum (Fovea pharyngea) fand ich bei 62,3 pCt. der Schädel.

Mit der Demonstration vorstehender Thatsachen hoffen wir bewiesen zu haben, dass in der Mitte des Nasenrachen- 
raumes sich eine Stelle befindet, die der Cmgebung gegenüber besondere anatomische Merkmale trägt. Dass diese Stelle sich als eine Grube, ein Recessus darstellt, wird durch das Verhalten des Os oceipitis mit seiner Massa fibrosa und der Mm. longi capitis veranlasst. Die Schleimhat trägt nichts weiter zur Formation dieser Grube bei, sie überzieht gleichmässig den Nasenrachenraum. Findet sich das adenoide Gewebe in der Mucosa stark entwickelt, so kann dem äusseren Ansehen nach die Grube ganz fehlen. Histologisch zeigt die Schleimhaut der Bursa pharyngea dieselbe Structur wie die der Umgebung.

Ob man der Mittelgrube des Nasenrachenraumes den Namen Bursa pharyngea oder Recessus pharyng. med. oder Fossa pharyng, med. giebt, halte ich für irrelevant.

Dass die Mittelgrube auch functionell besonders charakterisirt ist, konnte ich an einer von Herrn Professor Mikulicz wegen einer sarcomartigen Neubildung an Gesicht und Nase operirten Patientin demonstriren. Bei derselben waren die ganze äussere Nase, der linke Oberkiefer und die linken Nasenmuscheln entfernt worden, so dass der Nasenrachenraum auf's Bequemste übersehen werden konnte. Es zeigte sich nun, dass beim Schlucken der ganze Raum stark bewegt wird, nur die Mitte, wo die Schleimhaut sich tief zwischen den $\mathrm{Mm}$. longi capitis in die Massa fibrosa und den Knochen einbohrt, d. h. den Recessus medius bildet, sieht man unbewegt bleiben. Dass das so ist, kaun nach dem anatomischen Verhalten wohl kaum überraschen, und erscheint für die Einschleimung der Rachenwände von Wichtigkeit; ist doch durch die Unbeweglichkeit des Recessus medius ein Schleimreservoir geschaffen, aus dem nur allmählich, aber constant das Secret ausfliesst, so dass ein Trockenschlucken verhindert wird, was eintreten müsste, wenn der Recessus medius, ebenso wie die Recessus laterales (zwischen Tubenwulst und hinterer Rachenwand), beim Schluckact bewegt, comprimirt würde. Auf der anderen Seite ist aber in diesem physiologischen Verhalten auch wieder die Ursache für gewisse pathologische Vorkommnisse gegeben, ich meine für die Retention von Secret (Tornwaldt, Kafemann). Damit komme ich zu den patho- 
logischen Befunden, die ich über das Verhalten der Bursa pharyngea an der Leiche erheben konnte. Dieselben waren, wie oben schon erwähnt, ebenso dürftig wie einförmig. Nie habe ich eine nur im Rec. med. localisirte Tuberculose, Syphilis oder Diphtherie gesehen; war der Rec. med. derartig krank, so war es clie Umgebung mindestens eben so sehr. Die von Tornwald t in seiner Arbeit mehrfach erwähnte und am lebenden Individuum mir demonstrirte Cystenbildung der Bursa pharyngea habe ich an mehreren hundert Leichen, die ich im Danziger Stadtlazarett darauf hin untersuchte, sonderbarer Weise nie gefunden; recht häufig aber Secretionsanomalien, sowohl Hypersecretion normalen Schleimes, als auch intensive Eiterung bei intacter Umgebung. Aus den verschiedenen Crypten des Recessus medius konnte ich durch Druck mit dem Finger mehrere Centimeter lange Eiter-oder Schleimfäden entleeren, wie wir dieselben auch am Lebenden bei Pharynxkatarrhen oft $\mathrm{zu}$ beobachten Gelegenheit haben ( $\mathrm{K}$ afemann).

Durch vorstehende Auseinandersetzung wollte ich beweisen, dass Tornwaldt Recht hat, wenn er die sog. Bursa pharyngea als Einsenkung der Schleimhaut aufasst, und dass er Recht hat, wenn er an ihrer Stelle die häufigste und bedeutendste Localisation der krankbaft veränderten Secretion sucht.

Sollte dieser kleine Aufsatz Leser fiaden, so wird mancher vielleicht Literaturangaben und Citate vermissen. Absichtlich liess jch beides fort, um diese Mittheilung nicht überflüssiger Weise auszudehnen; bis zum Juli 1889 glaube ich über die zum Gegenstande gehörige Literatur ziemlich vollständig orientirt zu sein, und weiss ich daher auch, dass ich herzlich wenig Neues bringe.

Die Präparate, nach denen die Bilder gefertigt wurden, sind wohl noch alle in meinem Besitz und bin ich bereit, dieselben den sich dafür interessirenden gern zu zeigen, soweit es die äusseren VerhäItnisse irgend gestatten; ihre Herstellung ist übrigens ausserordentlich leicht. Wenn die erwähnten Thatsachen über das Verhalten der gesunden und kranken Bursa phar. so wenig bekannt und anerkannt worden sind, so lag das wohl einmal an dem wenig geschickt gewählten Namen "Bursa", dann aber vielleicht auch an dem Fehlen einer leichten Sections- 
methode des Nasenrachenraumes. Noch einmal möchte ich vor der Benutzung zu dicker Sügen warnen; dieselben können, zumal wenn es sich um Präparate von Kindern handelt, gar nicht dünn genug sein. Monate lang habe ich bei Neugebornen vergeblich nach der Schleimhanteinbohrung in der Mitte des Rachendaches gesucht und glaubte schon, dass dieselbe hier gar nicht existire, bis mein Freund, Dr. Ernst Friedländer in Danzig, sie mir als ganz dünnes, kaum sichtbares rothes Zäpfehen an einigen meiner Präparate zeigte. An anderen batte ich sie offenbar fortgesägt. Ich fand sie dann später immer, als ich Transversal. und Sagittalschnitte durch das kindliche Rachendach nur noch mit dem Messer machte. Also auch bei Kindern und Neugebornen giebt es eine Anlage zur Bursa pharyngea; ob sie beim Fötus vorkommt, nögen andere entscheiden.

\section{Erklärung der Abbildungen. Tafel III.}

a Stelle der sogenannten Bursa pharyngea. b Yassa fibrosa ossis occipitis. c Pars basilaris ossis occipitis. d M. longus capitis, in Fig. 2 von Schleimhaut bedeckt. e Hintere Rachenwand. f Septum narium. g Tubenmündung in Fig. 2, in Fig. 4 Tube selbst. 